\title{
TRATAMENTO TÉRMICO NA MANUTENÇÃO DA COLORAÇÃO DE LICHIAS ${ }^{1}$
}

\author{
ANGELA VACARO DE SOUZA², ROGÉRIO LOPES VIEITES ${ }^{3}$, \\ DOUGLAS SEIJUM KOHATSU ${ }^{4}$, GIUSEPPINA PACE PEREIRA LIMA ${ }^{5}$
}

RESUMO - O objetivo do trabalho foi avaliar a influência do tratamento térmico na coloração de frutos de lichia. Os frutos foram tratados por $0 ; 5 ; 10 ; 15 ; 20$ e 25 minutos de imersão em água a $45^{\circ} \mathrm{C}$, embalados em bandejas de poliestireno expandido e filme de policloreto de vinila $0,020 \mathrm{~mm}$ e armazenados em B.O.D. a $5^{\circ} \mathrm{C}$ e $90 \pm 5 \%$ de UR. O delineamento experimental foi em esquema inteiramente casualizado, com 6 tempos de armazenamento para as análises de coloração e enzimática, e até atingirem o limite de comercialização para a análise da vida útil, sendo os frutos analisados a cada 3 dias, com 3 repetições e 10 frutos por unidade experimental. Os parâmetros avaliados foram: análise visual do escurecimento, coloração e atividade específica da polifenol oxidase e da peroxidase. Os tratamentos usando 5 e 10 minutos de imersão foram os que apresentaram melhores resultados na manutenção da coloração dos frutos e na diminuição da atividade enzimática.

Termos para indexação: Litchi chinensis, escurecimento enzimático, cor.

\section{THERMAL TREATMENT IN LYCHEE COLOR MAINTENANCE}

ABSTRACT - The objective of the work was to evaluate the influence of the thermal treatment in the color of lychee fruits. The fruits were treated by $0 ; 5 ; 10 ; 15 ; 20$ and 25 minutes of water immersion at $45^{\circ} \mathrm{C}$, packaged in expanded polystyrene trays and film of polyvinyl chloride $0.020 \mathrm{~mm}$, stored in B.O.D. at $5^{\circ} \mathrm{C}$ and $90 \pm 5 \%$ of UR. The experimental delineation was in a completely randomized scheme, with 6 times of storage for the color and enzymatic analysis, until they reached the limit for the marketing for the analysis of the shelf-life, and the fruits were analyzed every 3 days, with 3 replications and 10 fruits in each experimental unity. The evaluated parameters were: visual analysis of the browning, color and specific activity of the polyphenol oxidase and the peroxidase. The treatments using 5 and 10 minutes of immersion showed the best results in maintaining the color of the fruits and in the reduction of the enzymatic activity.

Index terms: Litchi chinensis, enzymatic browning, color.

\section{INTRODUÇÃO}

A lichia é um fruto de alto valor comercial no mercado internacional. No entanto, perde rapidamente sua atrativa coloração vermelha após a colheita devido ao rápido escurecimento do pericarpo. $\mathrm{O}$ escurecimento reduz seu valor comercial e tem sido considerado o principal problema pós-colheita (Holcroft \& Mitcham, 1996). Isso se deve principalmente à degradação da antocianina em associação com a oxidação dos fenóis pelas enzimas polifenol oxidase (PPOs) e / ou peroxidase (POD) (Zhang et al., 2005).
A atividade das PPOs está estritamente ligada às mudanças de cor devido à formação de polímeros coloridos. Dessa forma, medidas de cor podem ser consideradas um índice indireto da atividade das PPOs e amostras que não apresentam acastanhamento ou outras cores anômalas são consideradas livres de PPO ativa (Severini et al., 2001). Outra hipótese para este escurecimento é o resultado da oxidação e polimerização de compostos fenólicos, incluindo das antocianinas, causado pela PPO e POD (Severini et al., 2001).

Swarts \& Anderson (1980) verificaram que curtos períodos de imersão em água quente podem

'(Trabalho 065-09). Recebido em: 16-03-2009. Aceito para publicação em: 25-11-2009.

${ }^{2}$ Mestre em Agronomia - Horticultura. Departamento de Produção Vegetal - Horticultura, Faculdade de Ciências Agronômicas/FCA/ UNESP - C.P. 237 - CEP 18.610-307-Botucatu-SP, Brasil.

avsouza@fca.unesp.br.

${ }^{3}$ Prof. Dr. Departamento de Produção Vegetal - Horticultura, Faculdade de Ciências Agronômicas/FCA/UNESP - C.P. 237 - CEP 18.610-307-Botucatu-SP, Brasil. vieites@fca.unesp.br.

${ }^{4}$ Doutorando em Agronomia - Horticultura. Departamento de Produção Vegetal - Horticultura, Faculdade de Ciências Agronômicas/ FCA/UNESP - C.P. 237 - CEP 18.610-307-Botucatu-SP, Brasil.

kohatsu@fca.unesp.br,

${ }^{5}$ Profa. Dra. Departamento de Química e Bioquímica, Instituto de Biociências/IBB/UNESP - C.P. 510 - CEP 18.618-000-BotucatuSP, Brasil.gpplima@ibb.unesp.br 
atrasar significativamente a taxa de desenvolvimento de podridão em lichias. Os tratamentos com água quente não só retardam o desenvolvimento do patógeno, mas também afetam a suscetibilidade dos frutos à infecção (Shirra et al., 2000).

Diversos trabalhos mostram a relação da temperatura de armazenamento e a manutenção da coloração de lichias. Olesen et al. (2003) verificaram que a temperatura ótima para o armazenamento das lichias, visando à retenção da cor vermelha do pericarpo, encontra-se entre 5 e $10^{\circ} \mathrm{C}$, mas, para controlar o desenvolvimento de podridões, esta temperatura se encontra-se entre 2 e $5^{\circ} \mathrm{C}$. Assim, o objetivo deste trabalho foi avaliar a influência do tratamento térmico na manutenção da vida pós-colheita através de medidas de coloração e da atividade enzimática de PPO e POD em lichias.

\section{MATERIAL E MÉTODOS}

\section{Aquisição dos frutos}

Foram utilizadas lichias (Litchi chinensis Sonn.) cv Bengal, no estágio de maturação fisiológica, provenientes de pomar comercial do Sítio Akamatsu (Carlópolis - Paraná, Brasil), latitude 23 $25^{\prime}$ 39'S, longitude $40^{\circ} 43^{\prime} 17^{\prime}$ e $550 \mathrm{~m}$ de altitude.

\section{Tratamentos e condições de armazena-} mento

Os frutos foram selecionados, lavados com água somente para a retirada de impurezas e o calor de campo e submetidos aos seguintes tratamentos: imersão dos frutos em água a $45^{\circ} \mathrm{C}$, por 5 minutos (T2); 10 minutos (T3); 15 minutos (T4); 20 minutos (T5); 25 minutos (T6) e o controle não foi submetido ao tratamento térmico (T1).

Depois de secos no ambiente, os frutos foram acondicionados em bandejas de poliestireno expandido e embalados com filme de policloreto de vinila $0,020 \mathrm{~mm}$, sendo que cada embalagem continha 10 frutos (cerca de 200 gramas) e armazenados em B.O.D. a $5^{\circ} \mathrm{C}$ e $90 \pm 5 \%$ de UR, segundo recomendação de Chitarra \& Chitarra (2005), por 15 dias, para as análises de coloração e enzimática, e até atingirem o limite de comercialização para a análise da vida útil (24 dias), sendo os frutos analisados a cada 3 dias.

\section{Escala de escurecimento}

$\mathrm{O}$ escurecimento foi analisado pelo número de dias em que os frutos se conservaram, em função da sua qualidade comercial. Para isso, foi utilizada uma escala de notas: 9 (frutos frescos e ausência de escurecimento); 7 (frutos frescos e leve escurecimento); 5 (pouco aspecto de frescor e moderado escurecimento); 3 (sem frescor e elevado escurecimento), e 0 (frutos desidratados e totalmente escurecidos), sendo que 5 foi a nota limite para a qualidade comercial.

\section{Coloração}

A coloração externa de 3 frutos foi realizada com a medição em dois lados de cada fruto, de cada repetição, utilizando-se de colorímetro Chroma meter (Minolta CR300), expressa pelo sistema com escala CIELAB ( $\left.\mathrm{L}^{*}, \mathrm{a}^{*}, \mathrm{~b}^{*}\right)$. Medições de coloração foram expressas em termos de valor $\mathrm{L}=$ luminosidade $[0$ (cor preta) a 100 (cor branca) $] ; \mathrm{a}=[-60,0$ (verde) a $+60,0$ (vermelho)]; segundo Olesen et al. (2003).

5. Atividade específica da Polifenol oxidase - PPO (EC. 1.14.18.1) e da Peroxidase - POD (EC 1.11.1.7)

Quinhentos miligramas (500mg) de casca foram pulverizados em $\mathrm{N}$ líquido, homogeneizados em $5 \mathrm{~mL}$ de tampão acetato de sódio $100 \mathrm{mM} \mathrm{pH}$ 5,0 e centrifugados a $12.000 \mathrm{~g}(10.000 \mathrm{rpm})$, por 25 minutos, a $4^{\circ} \mathrm{C}$, obtendo-se dessa maneira o extrato bruto. $\mathrm{O}$ sobrenadante foi utilizado para determinar a atividade da enzima peroxidase, segundo Lima et al. (1998), sendo a leitura feita a 505ๆm e expressa em $\mu \mathrm{mol} \mathrm{H}_{2} \mathrm{O}_{2}$ decomposto $\mathrm{g}^{-1}$ de $\mathrm{mg}$ proteína min $^{-1}$ e da enzima polifenol oxidase (Cano et al., 1997), lida a $395 \mathrm{\eta m}$. A atividade específica da enzima foi expressa em $\mu \mathrm{mol}$ catecol oxidado mg de proteína $^{-1}$ $\min ^{-1}$.

Para a medição da atividade específica das enzimas, foi feita a medição do teor total de proteína solúvel com a utilização do método de Bradford (1976).

\section{Delineamento experimental}

$\mathrm{O}$ delineamento experimental foi em esquema inteiramente casualizado, com 6 tempos de armazenamento: $(0 ; 3 ; 6 ; 9 ; 12$ e 15 dias $)$ para as análises de coloração e enzimática, e até atingirem o limite de comercialização para a análise da vida útil (24 dias), e 6 tratamentos (Controle; 5; 10; 15; 20 e 25 minutos de imersão em água a $45^{\circ} \mathrm{C}$ ) com 3 repetições e 10 frutos por unidade experimental. A análise estatística dos dados foi realizada utilizando o programa SISVAR (Ferreira, 2000). Para a comparação das médias, foi utilizado o teste de Tukey, a $5 \%$.

\section{RESULTADOS E DISCUSSÃO}

\section{Escala de escurecimento e coloração}

Para a exportação de lichia, há a necessidade de um período mínimo de uma a duas semanas; entretanto, a curta vida útil das lichias tem sido um empecilho significativo para este comércio (Underhill et al., 1997). O escurecimento enzimático do pericarpo da lichia é o primeiro sinal visível do declínio da qualidade da fruta. Todos os tratamentos 
mantiveram a qualidade comercial dos frutos até o $15^{\circ}$ dia de análise. A partir do $18^{\circ}$, os frutos dos tratamentos 4 ; 5 e $6(15 ; 20$ e 25 minutos de imersão, respectivamente) foram descartados devido à baixa qualidade. O tratamento 3 (10 minutos de imersão) foi o que apresentou maior manutenção na qualidade dos frutos (Figura 1), seguido pelo tratamento 2 (5 minutos).

Os melhores tratamentos para a manutenção dos valores de L (Figura 2) foram o uso de 5 e 10 minutos de imersão em água a $45^{\circ} \mathrm{C}$. Os tratamentos que foram submetidos por 15; 20 e 25 minutos, apresentaram diminuição significativa nos valores deste parâmetro. Dados semelhantes foram encontrados por Olesen et al. (2004), que estudaram a influência do binômio tempo $\mathrm{X}$ temperatura na manutenção da qualidade de lichia cv. 'Kwai May Pink', armazenada a $5^{\circ} \mathrm{C}$ e observaram que, 19 dias após a colheita, os frutos imersos por 5 minutos a $52^{\circ} \mathrm{C}$ e 2 minutos a $50^{\circ} \mathrm{C}$ apresentavam valores de $\mathrm{L}$ superiores aos frutos- controle.

Diminuição nos valores de L em lichia foi relatada por Saengnil et al. (2006), que estudaram a influência da imersão dos frutos de lichia em água quente $\left(98^{\circ} \mathrm{C}\right.$ por $\left.30 \mathrm{~s}\right)$, e de imersões em soluções de ácido oxálico, ácido cítrico e ácido ascórbico a $0 ; 2,5 ; 5 ; 10$ e $15 \%$, por 15 minutos, e observaram que a imersão em água quente diminuiu os valores de L, deixando os frutos mais escuros. Isso pode ser devido à alta temperatura utilizada e um possível escurecimento não enzimático.

A imersão de lichia em água à temperatura de $55^{\circ} \mathrm{C}$, por 20 segundos, aumenta sua vida pós-colheita (Fallik, 2004). Por outro lado, o uso do tratamento térmico promoveu a diminuição nos valores do parâmetro 'a' em frutos de lichia cv. 'Hong Huay' (Saengnil et al., 2006). Os valores de 'a' apresentaram pouca variação ao longo do tempo de armazenamento nos tratamentos $1 ; 2$ e 3 , enquanto os demais tratamentos apresentaram diminuição nos valores deste parâmetro, mostrando que o alto tempo de exposição ao tratamento causou degradação dos pigmentos da casca (Figura 3). Zhang et al. (2005) observaram que os níveis do pigmento antocianina diminuem com o aumento do escurecimento e com o aumento do tempo de armazenamento. Assim, neste trabalho, pode ser observada a diminuição do escurecimento com o tratamento térmico, principalmente 5 e 10 minutos, o que poderia ter afetado o metabolismo de degradação da antocianina.

Dados semelhantes foram encontrados por Olesen et al. (2004), que verificaram que os frutos submetidos a $50^{\circ} \mathrm{C}$, por 2 minutos, apresentaram resultados superiores ao controle, confirmando a eficácia do tratamento térmico. A utilização do tratamento hidrotérmico, por pelo menos 2 minutos a $50^{\circ} \mathrm{C}$, descrita por Wong et al. (1991) e Underhill \& Critchley (1993), indicou que lichias têm seu escurecimento diminuído.

Recentemente, um procedimento com spray de vapor de água foi desenvolvido por Lichter et al. (2000) para o tratamento da fruta. As frutas tratadas por este procedimento podem ser armazenadas durante, pelo menos, 35 dias armazenadas sob refrigeração, mantendo-se uma cor vermelha uniforme.

\section{Atividade específica da polifenol oxidase e da peroxidase}

Os dados apresentados na Figura 4 mostram que o tratamento térmico atuou na diminuição da atividade da enzima PPO assim que os frutos foram tratados. Esta diminuição pode ter sido responsável pelo menor escurecimento nos frutos. De acordo com Jiang et al. (1996), o tratamento térmico a $55^{\circ} \mathrm{C}$, durante 2 min, diminui a atividade da enzima PPO, o que pode auxiliar na diminuição do escurecimento dos frutos de lichia.

Os frutos submetidos a 20 e 25 minutos em água a $45^{\circ} \mathrm{C}$ apresentaram maior inibição na atividade, porém apresentaram maior escurecimento. Isso pode ser explicado pelo alto tempo de imersão dos frutos, que pode ter ocasionado a caramelização dos açúcares presentes na casca (reação de Maillard). Lichias tratadas com água quente à temperatura de $98^{\circ} \mathrm{C}$, por 30 segundos, apresentaram diminuição na atividade da enzima PPO em frutos armazenados por 5 dias a $25^{\circ} \mathrm{C}$ (Saengnil et al., 2006).

A POD apresentou comportamento semelhante ao da PPO, com diminuição da atividade logo após o tratamento e aumento da atividade com o passar do período de armazenamento (Figura 5). Zheng \& Tian (2006) encontraram resultados semelhantes quando utilizaram diferentes concentrações de ácido oxálico na atividade das enzimas POD e PPO, e concluíram que, após a imersão, a atividade enzimática foi menor que as do fruto- controle. Maiores atividades da POD e PPO foram encontrados por Zhang et al. (2005) em frutos sem tratamento, durante 6 dias de armazenamento. A atividade da POD aumenta consistentemente com o índice de escurecimento, mas negativamente com a concentração de antocianina durante o armazenamento da lichia. 


\section{Vida útil}

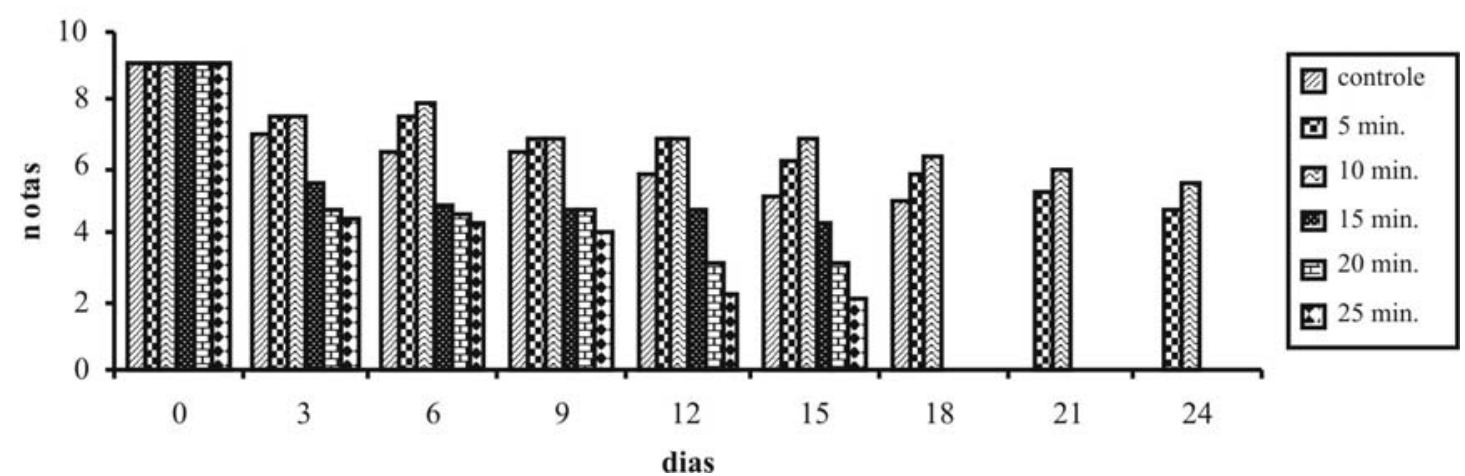

FIGURA 1 - Valores médios de escurecimento (escala de notas): 9 (frutos frescos e ausência de escurecimento); 7 (frutos frescos e leve escurecimento); 5 (pouco aspecto de frescor e moderado escurecimento); 3 (sem frescor e elevado recurecimento), 0 (frutos desidratados e totalmente escurecidos), sendo que 5 foi a nota limite para a qualidade comercial em frutos de lichia, em função dos tratamentos e das épocas de análise.

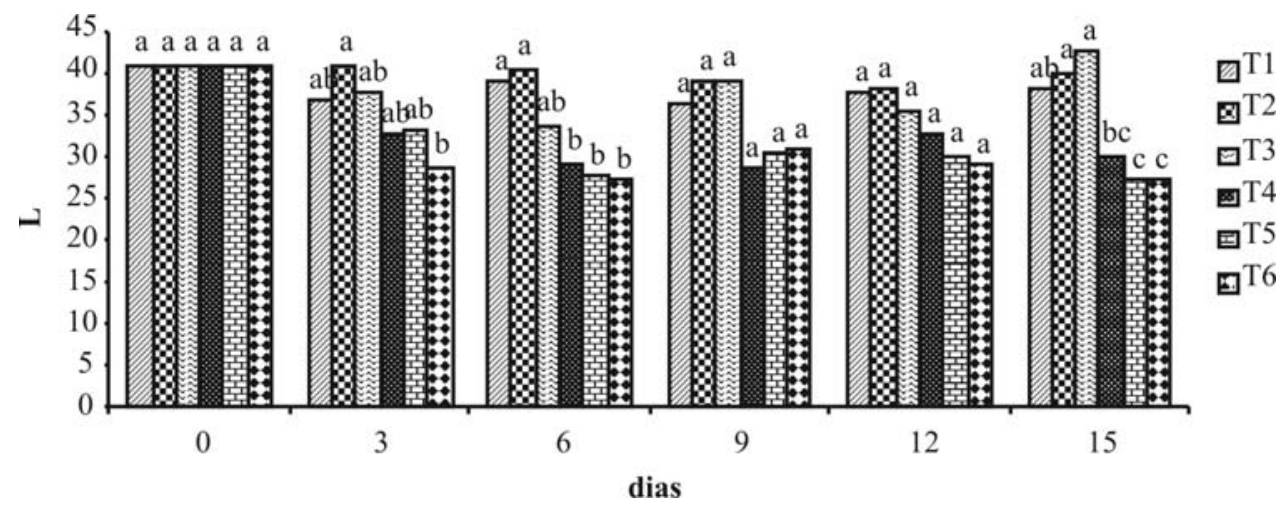

FIGURA 2 - Valores médios de coloração - L (Luminosidade) em frutos de lichia, em função dos tratamentos e das épocas de análise. $(\mathrm{T} 1=$ Controle; $\mathrm{T} 2=5$ minutos $; \mathrm{T} 3=10$ minutos; $\mathrm{T} 4=15$ minutos; $\mathrm{T} 5=20$ minutos e T6 = 25 minutos ).Médias seguidas de mesma letra não diferem entre si, pelo Teste de Tukey, a 5\% de probabilidade.

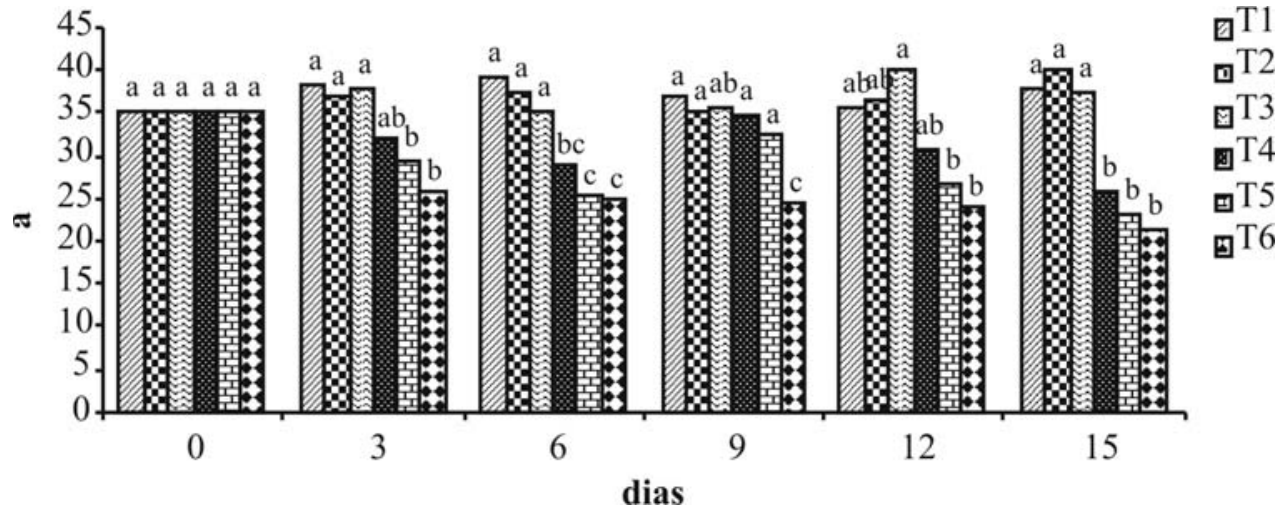

FIGURA 3 - Valores médios de coloração - 'a' em frutos de lichia, em função dos tratamentos e das épocas de análise. $(\mathrm{T} 1=$ Controle; $\mathrm{T} 2=5$ minutos; $\mathrm{T} 3=10$ minutos; $\mathrm{T} 4=15$ minutos; $\mathrm{T} 5=20 \mathrm{mi}-$ nutos e T6 $=25$ minutos). Médias seguidas de mesma letra não diferem entre si, pelo Teste de Tukey, a $5 \%$ de probabilidade. 


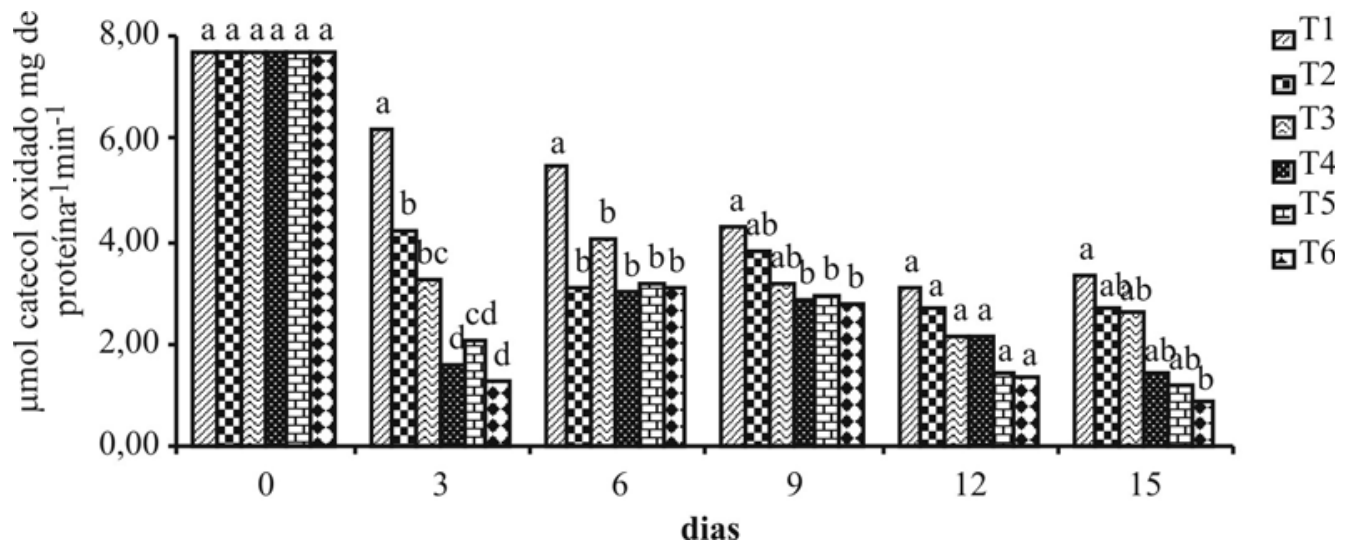

FIGURA 4 - Valores médios da atividade enzimática específica - PPO ( $\mu$ mol catecol oxidado mg de proteína ${ }^{-1} \mathrm{~min}^{-1}$ ) em frutos de lichia, em função dos tratamentos e das épocas de análise. (T1 = Controle; $\mathrm{T} 2=5$ minutos; $\mathrm{T} 3=10$ minutos; $\mathrm{T} 4=15$ minutos; $\mathrm{T} 5=20$ minutos e $\mathrm{T} 6=25$ minutos ).Médias seguidas de mesma letra não diferem entre si, pelo Teste de Tukey, a 5\% de probabilidade.

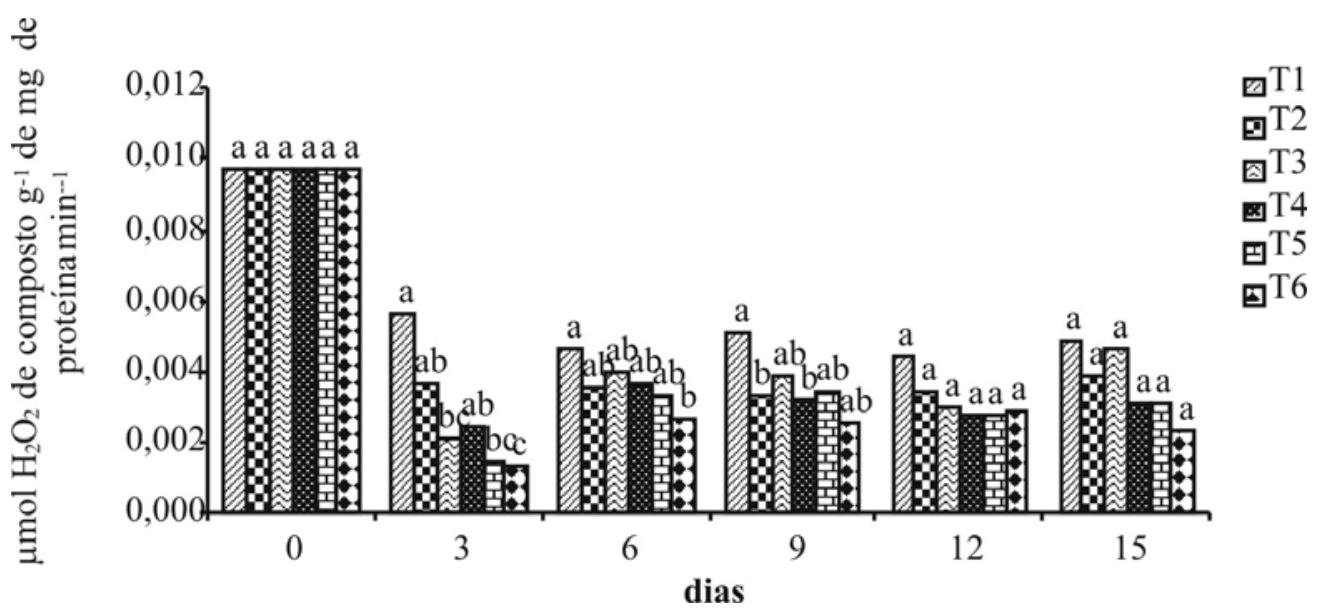

FIGURA 5 - Valores médios da atividade enzimática específica - POD $\left(\mu \mathrm{mol} \mathrm{H}_{2} \mathrm{O}_{2}\right.$ decomposto $\mathrm{g}^{-1}$ de $\mathrm{mg}$ proteína $\min ^{-1}$ ) em frutos de lichia, em função dos tratamentos e das épocas de análise. (T1 $=$ Controle; $\mathrm{T} 2=5$ minutos ; $\mathrm{T} 3=10$ minutos; $\mathrm{T} 4=15$ minutos; $\mathrm{T} 5=20$ minutos e $\mathrm{T} 6=25$ minutos ).Médias seguidas de mesma letra não diferem entre si, pelo Teste de Tukey, a 5\% de probabilidade. 


\section{CONCLUSÃO}

1-O tratamento térmico, quando aplicado por 5 e 10 minutos a $45^{\circ} \mathrm{C}$, mostrou-se eficaz na manutenção da coloração dos frutos. As enzimas POD e PPO podem ser consideradas marcadores bioquímicos de escurecimento em lichia.

2-O tratamento térmico com 10 minutos de imersão favoreceu o possível aumento no tempo de comercialização devido ao prolongamento da vida pós-colheita, sendo que a fruta possui safra muito curta e alto preço. $\mathrm{O}$ aumento no número de dias para a comercialização pode garantir ganhos significativos aos produtores, sendo o tratamento térmico uma tecnologia barata.

\section{AGRADECIMENTOS}

Ao CNPq, pelo suporte financeiro.

\section{REFERÊNCIAS}

BRADFORD, M. M. A rapid and sensitive method for the quantification of microgram quantities of proteins utilizing the principle-dye binding. Analytical Biochemistry, Orlando, v.72, p.248-254, 1976.

CANO,M. P.; DE ANCOS, B.; CRUZ MATALLANA, M.; CAMARA, M.; REGLERO, G.; TABERA, J. Differences among Spanish and LatinAmerican banana cultivars: morphological, chemical and sensory characteristics. Food Chemistry, Kidlington, v.59, p.411-419, 1997.

CHITARRA, M. I. F.; CHITARRA, A. B. Pós-colheita de frutos e hortaliças: fisiologia e manuseio. Lavras: UFLA, 2005. 785p.

FALLIK, E. Prestorage hot water treatments (immersion, rinsing and brushing). Postharvest Biology and Technology, Amsterdan, v.32, p.125-134, 2004.

FERREIRA, D.F. Análises estatísticas por meio do Sisvar para windows versão 4.0. In: REUNIÃO ANUAL DA REGIÃO BRASILEIRA DA SOCIEDADE INTERNACIONAL DE BIOMETRIA, 45 , 2000, São Carlos. Anais... São Carlos: Universidade Federal de São Carlos, 2000. p.255-258.
HOLCROFT, D. M.; MITCHAM, E. J. Review: postharvest physiology and handling of litchi (Litchi chinensis Sonn.). Postharvest Biology and Technology, Amsterdam, v.9, n.1, p. 265-281, 1996.

JIANG, Y.M.; LIU, S.X.; LI, Y.B.; CHEN, F. A reassessment of heat treatment as a means of reducing the occurrence of browning of litchi (Litchi chinensis Sonn.) fruit. International Journal of Tropical Agriculture, Hisar, v.14, p.163-167, 1996.

LICHTER, A.; DVIR, O.; ROT, I.; AKERMAN, M.; REGEV, R.; WIESBLUM, A. FALLIK, E.; ZAUBERMAN, G.; FUCHS, Y. Hot water brushing: an alternative method to $\mathrm{SO} 2$ fumigation for color retention of litchi fruits. Postharvest Biology and Technology, Amsterdam, v.18, p.235-244, 2000.

LIMA, G.P.P.; BRASIL, O.G.; OLIVEIRA, A. M. Poliaminas e atividade da peroxidase em feijão (Phaseolus vulgaris L.) cultivado sob estresse salino. Scientia Agrícola, Piracicaba, v.56, n.1, p.21-25, 1998.

OLESEN, T.; NACEY, L.; WILTSHIRE, N.; O'BRIEN, S. Hot water treatments for the control of rots on harvested litchi (Litchi chinensis Sonn.) fruit. Postharvest Biology and Technology, Amsterdam, v.32, p.135-146, 2004.

OLESEN, T.; WILTSHIRE, N.; MCCONCHIE, C. Improved post-harvest handling of lychee. Queensland: Rural Industries Research and Development Corporation, 2003. 86p.

SAENGNIL, K.; LUEANGPRASERT, K.; UTHAIBUTRA, J. Control of enzymatic browning of harvested 'Hong Huay' litchi fruit with hot water and oxalic acid dips ScienceAsia, Shangai, v.32, p.345-350, 2006.

SEVERINI, C.; PILLI, T. de; BAIANO, A.; MASTROCOLA, D.; MASSINI, R. Preventing enzymatic browning of potato by microwave blanching. Sciences des Aliments, Paris, v. 21, p. 149-160, 2001.

SHIRRA, M.; D'HALLEWIN, G.; BEN-YEHOSHUA, S.; FALLIK, E. Host-pathogen interactions modulated by heat treatment. Postharvest Biology and Technology, Amsterdam, v.21, p.71-85, 2000. 
SWARTS, D.H.; ANDERSON, T. Chemical control of mould growth on litchis during storage and sea shipment. Subtropica,v.1, n.10, p.13-15, 1980.

UNDERHILL, S.J.R.; COATES, L.M.; SAKS, Y. Litchi. In: MITRA, S.K. (Ed.). Postharvest physiology and storage of tropical and subtropical fruits. London: CAB International, 1997. p.191-208.

UNDERHILL, S. J. R.; CRITCHLEY, C. Physiologycal, biochemical and anatomical changes in lychee (Litchi chinensis Soon.) pericarp during storage. Journal of Horticultural Science, Ashford, v.68, p.327-335, 1993.
WONG, L.S.; JACOBI, K.K.; GILES, J.E. The influence of hot benomyl dips on the appearance of cool stored lychee (Litchi chinensis). Scientia Hortikculture, Amsterdam, v.46, p.245-251, 1991.

ZHANG, Z.; PANG, X.; XUEWU, D.; JI, Z.; JIANG, $Y$. Role of peroxidase in anthocyanin degradation in litchi fruit pericarp. Food Chemistry, Kidlington, v.90, p.47-52, 2005.

ZHENG, X.; TIAN, S. Effect of oxalic acid on control of postharvest browning of litchi fruit. Food Chemistry, Kidlington, v.96, p.519-523, 2006. 